\title{
Simulation and performance analysis for coefficient estimation for sinusodial signal using LMS, RLS and proposed method
}

\author{
Kantipudi MVV Prasad ${ }^{1}$, Dr. H.N. Suresh ${ }^{2}$ \\ ${ }^{1}$ Research Scholar, Visvesvaraya Technological University, Belgaum, India \\ ${ }^{2}$ Department of Instrumentation, Bangalore Institute Technology, Bangalore, India \\ *Corresponding author E-mail: prasadb2016@gmail.com
}

\begin{abstract}
The estimated Power Spectral Density (PSD) gives the information regarding the architectural structure of random process; it can be utilized for mathematical modeling, removal of noise, prediction of the signal of the deserved processes. The objective of spectral density estimation is to approximation the spectral density of a random signal from a series of time sample of the signal. Spectral estimation and coefficient estimation is concerned with determining the distribution in frequency of the power of a random process. In this paper, a wellknown adaptive filter is used to the estimation of the spectral density of the signal. It includes the LMS, RLS and improves RLS (proposed method) to analyze the coefficient of the sinusoidal signal.
\end{abstract}

Keywords: Spectral Estimation; Coefficient Estimation; LMS; RLS; Improved RLS; Power Spectral Design; PDF.

\section{Introduction}

The power spectral evaluation of cyclic and arbitrary signals is one of the most vital appliance areas of DSP. Spectral estimation then becomes a problem of estimating the parameters in the assumed scheme. If this scheme is not fit the data, the parametric model performance will degrade, and lead to a biased estimation [1]. The single channel PSD estimation; there are two broad categories of PSD estimators. Among them, the Fourier-based estimators are the most popular. Also, the Capon and Amplitude and Phase estimate with much lower side lobes and more narrow spectral peaks than the fast Fourier transform methods, which is also a special case of Finite Impulse Response (FIR) filtering approaches.

To overcome these disadvantages, an alternative estimation procedure for data with a mixed spectrum containing an unknown number of spectral lines was proposed. An adaptive equalizer is customarily placed in the receiver with the channel output as the source of excitation applied to the equalizer; different parameters are adjusted using or Recursive least squares (RLS) algorithm to provide an estimate of each symbol transmitted. The drawbacks of the RLS algorithm, the increased computational power required and the output is unsteadiness. So, to get better the performance of RLS algorithm, the adaptive capon and amplitude and phase estimation based spectral analysis methods are used.

Regarding the computational complexity, conventional coefficients evaluation scheme, like LS, RLS and LMS cannot achieve fast convergence speed and little computational complexity at the same time. LS algorithm works on a block of data samples and requires a matrix inversion. RLS and LMS algorithms are updated sample by sample. RLS algorithms converge rapidly but consume a group of hardware resources. LMS algorithm consumes little hardware resources but converges slowly. By applying the orthonormal basis functions, we recommend an adaptive method that exhibits a low computation complexity of LMS algorithm while retaining the very fastly convergence velocity of RLS algorithm.

The remaining part of the manuscript is planned as follows: Section 2 describes the specific work done by other authors on an adaptive DPD system and then Section 3 proposes a general approach to derive the orthonormal basis functions that can alleviate the numerical instability during the coefficients estimation. And also includes the implementation of the model coefficients estimation algorithms. We propose an adaptive algorithm by applying the orthonormal basis functions. Section 4 validates the efficiency of the projected algorithm via simulation done in Matlab with experimental results. At the end of this paper is concluded with the conclusion of this projected method.

\section{Literature survey}

This section briefly summarizes related works done by the different author based on orthonormal basis function that can alleviate the numerical instability during the coefficients estimations.

Ram et al. [1] employed in critical care units to measure the vital medical parameters like heart speed and blood infiltration levels. In this work, we present an adaptive coefficient estimation technique to detect motion artifact (MA) components from quasiperiodic natured photoplethysmographic (PPG) signal and then deduce MA reduced PPG signal. Fourier coefficients are estimated using basic least mean squares algorithm. The novelty of the proposed technique lies in detection and reduction of MA noise by estimating the Fourier coefficients and then based on randomness measures considering only the required number of Fourier coefficients dynamically to generate MA reduced PPG signal. $\mathrm{SpO} 2$ has estimated from MA reduced PPGs by utilizing the calibration curve. The superiority of proposed technique is proved by comparing the experimental results with results obtained using basic least mean squares (LMS) method. PPG data recorded with 
different MA (Vertical, Horizontal and Bending movements of patient's finger) is considered for experiment analysis. Obtained $\mathrm{SpO} 2$ parameter calculations proved the efficacy of estimation technique in the measurement of reliable and accurate $\mathrm{SpO}$, helpful for medical diagnosis.

Choi et al. [2] presented an efficient method like linear zed RLS method, in which it utilize the both vehicle lateral as well as longitudinal dynamics in real time. The study indicates that the evaluated algorithm was examined through vehicle dynamic software Carsim as well as Matlab tool. Sardellitti et al. [3] proposed a distributed recursive least squares (RLS) algorithm incorporating an 11-norm regularization with time-varying regularization coefficient that enables a recursive distributed solution with no losses on the centralized solution. The method is especially useful in cooperative sensing when the parameters to be estimated are structurally sparse and time-varying. As well known, the 11-norm is useful to recover sparsity, but it also introduces a non-negligible bias. To tackle this issue, we further apply a garrote correction to our distributed mechanism that strongly reduces the bias.

Kobayashi et al. [4] accessible a simple system of combination coefficient evaluation with RLS filter is projected. Dynamics of WPT scheme is analyzed with a transfer function. Furthermore, the modelling and the manage technique of DC/DC converter are introduced. The investigational outcome shows that the coupling coefficient evaluation is provided the effective of the proposed system and it is controlled in a real-time. Wanger et al. [5] studied Diffusion strategies for learning across networks which minimize the transient regime mean-square deviation across all nodes is presented. The problem of choosing combination coefficients which minimize the mean-square deviation at all given time instances results in a quadratic program with linear constraints The implementation of the optimal procedure is based on the estimation of weight deviation vectors for which an algorithm is proposed. Additionally, the optimization that uses relaxed constraints is considered. The results show a potential for significant improvement of the convergence speed.

Kobayashi et al. [6] explaining the efficiency is controlled by exchanging the input impedance at the receiving end of the converter. But the proposed method has not at all been useful in the sequence of coupling coefficient is desirable continually to attain the control. In this paper, an easy technique of coupling coefficient evaluation with RLS filter and improve efficiency control using a PID feedback controller are planned.

Umay et al. [7] propose a new adaptive biomedical capsule localization scheme utilizing a recent geometric cooperative sensor technique to estimate the path loss coefficient for permittivity for time-of-flight (TOF) based range sensors. This cooperative environmental coefficient estimation technique involves the use of a mobile sensor triplet in place of a single sensor, and provides instantaneous estimates. K. Dongarkar et al. [8] proposes an online estimation strategy to simultaneously estimate the vehicle mass, road frictional coefficient and wind velocity for a Series-Parallel Hybrid vehicle. The estimation strategy uses inputs from the vehicle longitudinal accelerometer sensor for determining road grade along with other powertrain signals.

Das et al. [9] introduced a new sparsity-aware RLS method is implemented for distributed learning in a diffusion network. The algorithm deploys a RLS based adaptive filter at each node which is made sparsity-aware by regularizing the conventional RLS cost function with a sparsity promoting penalty. The regularization introduces certain "zero-attracting" terms in the RLS update equation which help in shrinkage of the coefficients. Each node shares its tap weight information with every other node in its neighborhood and refines its estimate by linearly combining the incoming tap weight information from neighboring nodes by a set of pre-defined weights. Results on both first and second order convergence of the algorithm are also provided. As simulations show, the proposed scheme outperforms additionally obtainable algorithms together in conditions of convergence speed and steady-state excess mean square error.

\section{Proposed method and implementation}

This division talks about the computational complexity of RLS algorithm, LMS algorithm, and the proposed algorithm. In this paper, we only compare the number of real multiplications required by each algorithm per iteration. Fig 1 shows the system architecture of the proposed method. It contains the input signal generation block. It generates the sinusoidal signal for this proposed method to get the PDF as well as PSD and coefficient estimation of these proposed methods. The orthonormal basis function constant is selected for this simulation work. The quantization will be performed. The error PDF will be calculated after this step. Finally, the constellation M-ary is initialized like $4,16,32,64,128$ and 256 are selected. At the end of this system, the coefficient will be estimated for all the techniques and also the comparison is performed.

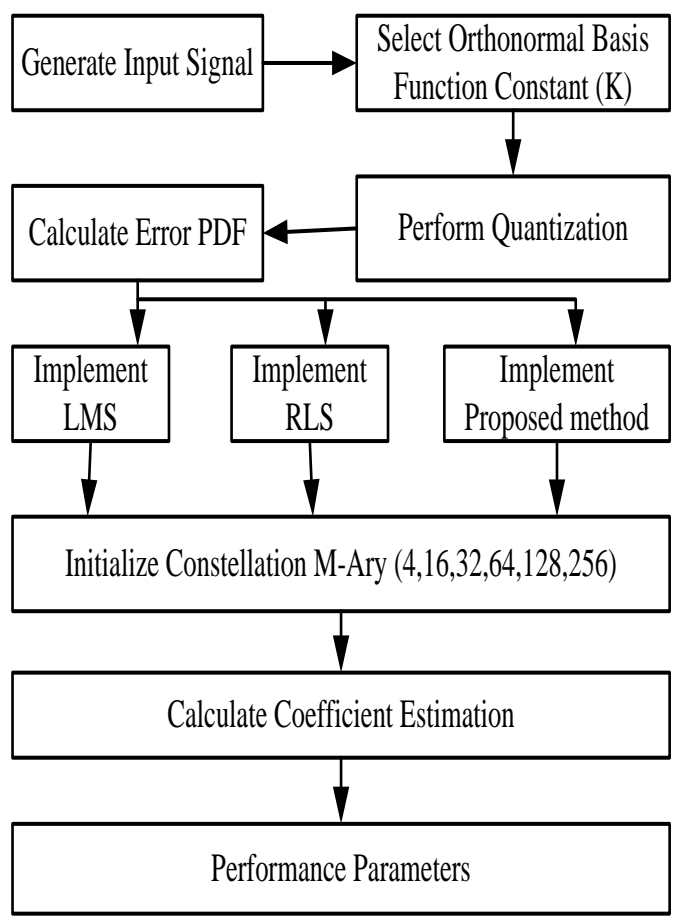

Fig. 1: System Architecture of the Proposed Method.

The projected technique is implementing by means of three different algorithms like LMS, RLS and finally the proposed method. The following section gives the pseudo code for the postposed method as well as another method.

Pseudo Code for cost effective LMS:

1) Start

2) Initialize Xbit, $M$, mod;

3) Calculate k, Nbit, Dtotal, Nframe;

4) Initialize Dsam $=64, \mathrm{Dfft}=128, \mathrm{Dgi}=22, \mathrm{Stotal}=150$;

5) Perform QAM/PSK 16-bit modulation;

6) Transmit signal;

7) Xint $\leftarrow$ Convert the bits in Xbit into k-bit symbols;

8) $\mathrm{A} 1 \leftarrow$ modulate using $16-\mathrm{QAM}$;

9) For 1:Nframe

10) \{

11) A2 $\leftarrow$ Initialize vector;

12) Extract data samples form $1^{\text {st }}$ frame;

13) Perform zero padding;

14) B1 $\leftarrow$ Perform IFFT operation on data;

15) For $\mathrm{i}=1: \mathrm{Dfft}$

16) \{

17) Arrange IFFT data samples one by one;

18) $\mathrm{B} 2 \leftarrow$ Generate data streams; 
19) $\}$

20) $\}$

21) End:

22) End;

23) Add cyclic prefix;

24) Add Gaussian noise;

25) End;

Pseudo Code for cost effective Proposed Method:

1) Start

2) Input Xbit, M, mod;

3) Calculate k, Nbit, Dtotal, Nframe;

4) Initialize Dsam=64,Dfft=128,Dgi=22;

5) Stotal $=\mathrm{Dfft}+\mathrm{Dgi} ; \%$ Total symbol length with guard interval;

6) Perform QAM/PSK 16-bit modulation;

7) Transmit signal;

8) Xint $\leftarrow$ Convert the bits in Xbit into k-bit symbols;

9) $\mathrm{A} 1 \leftarrow$ modulate using $16-\mathrm{QAM}$;

10) Perform IFFT operation;

11) Perform Orthonormal to each other;

12) Add Gaussian noise;

13) End;

Pseudo Code for cost effective RLS:

1) Start

2) Input Xbit, M, mod, $\mathrm{h}[]$;

3) Initialize $\mathrm{N}=2000$, sysorder $=10$;

4) Perform training;

5) Lambda $=0.9995$

6) Initialize $P$ matrix;

7) $\mathrm{P}=$ delta * eye (sysorder );

8) $\quad w=$ zeros $($ sysorder, 1$)$;

9) for $\mathrm{n}=\operatorname{sysorder}: \mathrm{N}$

10) $\mathrm{u}=\operatorname{inp}(\mathrm{n}:-1: \mathrm{n}-\mathrm{sys}$ sorder+1) ;

11) $\mathrm{phi}=\mathrm{u}^{\prime} * \mathrm{P}$;

12) $\mathrm{k}=$ phi'/(lamda + phi $* \mathrm{u})$;

13) $y(n)=w^{\prime} * u$;

14) $\mathrm{e}(\mathrm{n})=\mathrm{d}(\mathrm{n})-\mathrm{y}(\mathrm{n})$;

15) $\mathrm{w}=\mathrm{w}+\mathrm{k} * \mathrm{e}(\mathrm{n})$;

16) $\mathrm{P}=(\mathrm{P}-\mathrm{k} *$ phi $) /$ lamda ;

17) \% Just for plotting

18) $\operatorname{Recordedw}(1:$ sysorder, $n)=w$;

19) end

20) for $n=N+1:$ totallength

21) $\mathrm{u}=\operatorname{inp}(\mathrm{n}:-1: \mathrm{n}-\mathrm{sys}$ order+1) ;

22) $y(n)=w^{\prime} * u$;

23) $e(n)=d(n)-y(n)$;

24) end

25) Initialize Dsam $=64, \mathrm{Dfft}=128, \mathrm{Dgi}=22, \mathrm{Stotal}=150$;

26) Calculate k, Nbit, Dtotal, Nframe;

27) Perform QAM/PSK 16-bit modulation;

28) Transmit signal;

29) Xint $\leftarrow$ Convert the bits in Xbit into k-bit symbols;

30) $\mathrm{A} 1 \leftarrow$ modulate using $16-\mathrm{QAM}$;

31) For 1:Nframe

32) \{

33) A2 $\leftarrow$ Initialize vector;

34) Extract data samples form 1st frame;

35) Perform zero padding;

36) B1 $\leftarrow$ Perform IFFT operation on data;

37) For $i=1: D f f t$

38) \{

39) Arrange IFFT data samples one by one;

40) $\mathrm{B} 2 \leftarrow$ Generate data streams;

41) \}

42) $\}$

43) End:

44) End;

45) Add cyclic prefix;

46) Add Gaussian noise

47) End;
Table 1: Notations

\begin{tabular}{lll}
\hline 1. & Xbit & Input binary data \\
\hline 2. & M & M-ary \\
3. & mod & Modulation techniques (QAM/PSK) \\
4. & B3 & OFDMA signal \\
5. & A1 & Modulation signal after modulation \\
6. & k & Number of bits per symbol \\
7. & Nbit & Nbit=1024*k; \\
8. & Dtotal & All transmitted data symbol \\
9. & Dsam & 64 samples of data per frame \\
10. & Dfft & 128 samples per frame for IFFT \\
11. & Dgi & length of guard interval \\
12. & Stotal & Total symbol length with guard interval. \\
\hline
\end{tabular}

\section{Research and discussion}

In order to validate the effectiveness of the proposed lowcomplexity predistortion algorithm in terms of spectral regrowth suppression, experiments are performed to calculate the PDF, PSD and coefficient estimations of the system. Fig 2 to fig 12 shows the outcomes of the proposed method. In the method we assumed orthonormal basis function constant as 2 . It gives the quantization of the signal. Error vector signal also displayed in this paper. The error PDF of signal of $\mathrm{k}=1,2,3,4$ and 5 also displayed.

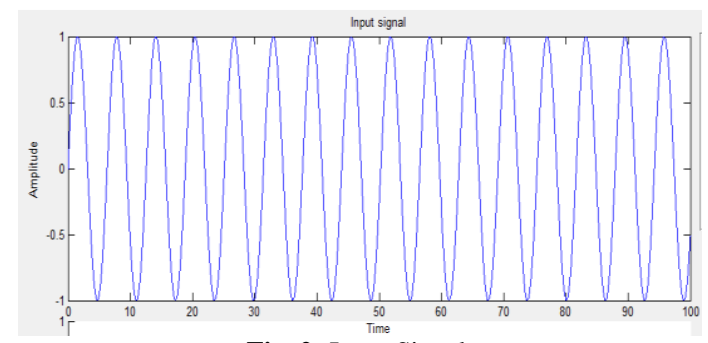

Fig. 2: Input Signal.

Select Orthonormal Basis Function Constant $(\mathrm{K})($ range 1-5) $=2$.

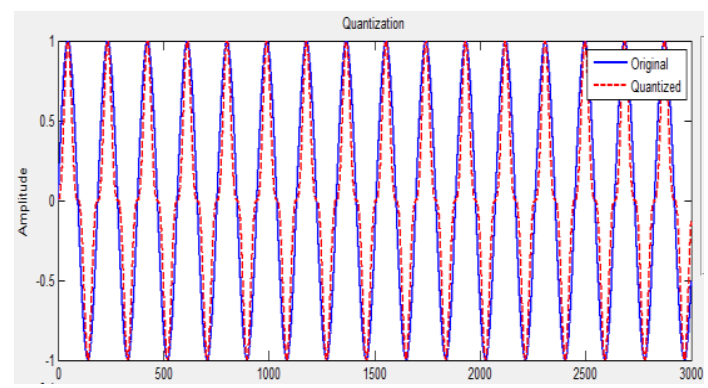

Fig. 3: Quantization of Signal.

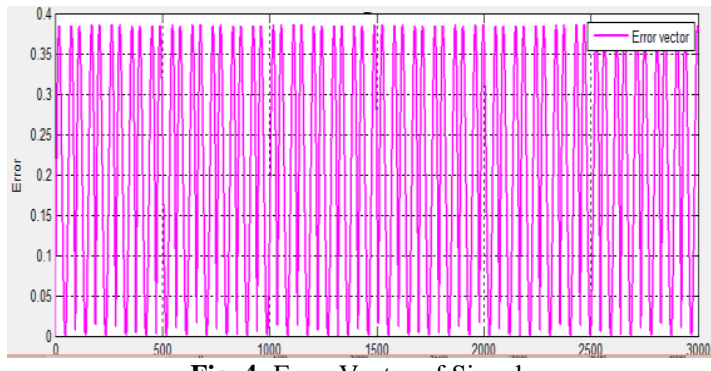

Fig. 4: Error Vector of Signal. 


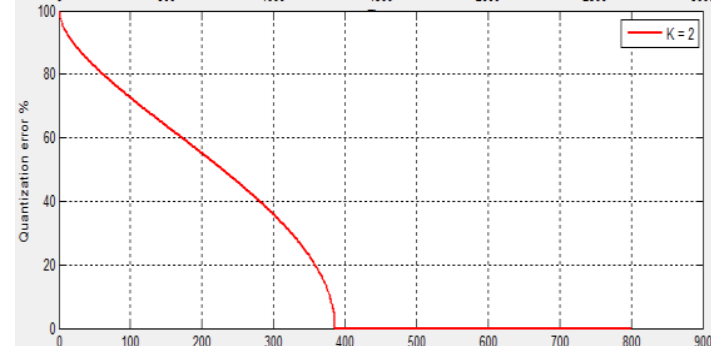

Fig. 5: Error PDF of Signal For $K=2$.

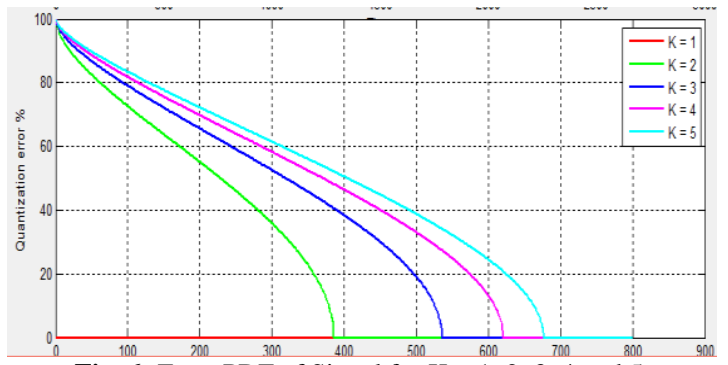

Fig. 6: Error PDF of Signal for $\mathrm{K}=1,2,3,4$ and 5 .

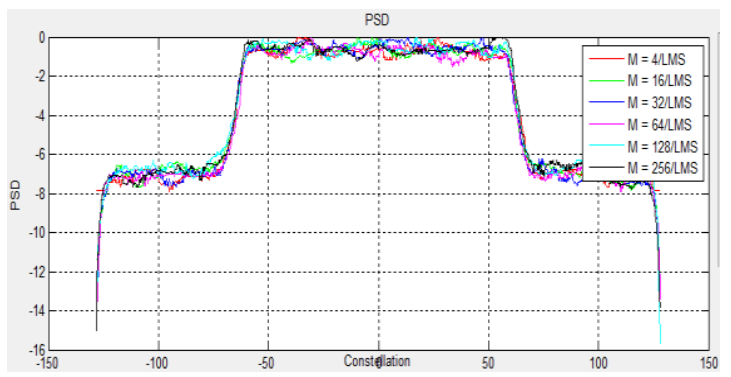

Fig. 7: Coefficient Estimation (PDF) For LMS Algorithm for Different Values of Constellation M-Ary=4, 16, 32, 64, 128, 256.

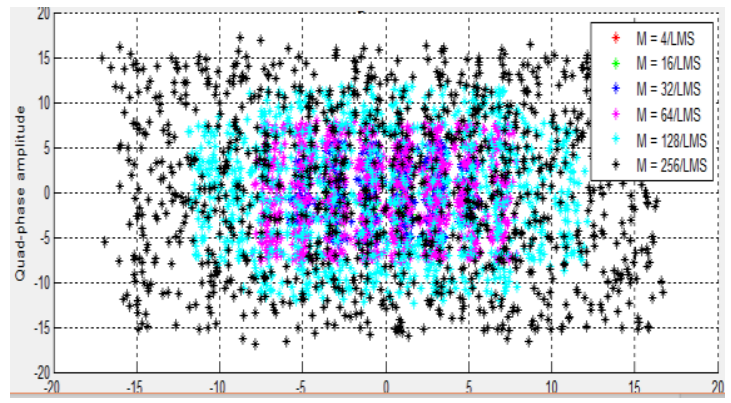

Fig. 8: Coefficient Estimation (Quad-Phase Amplitude) for LMS Algorithm for Different Values of Constellation M-Ary=4, 16, 32, 64, 128, 256.

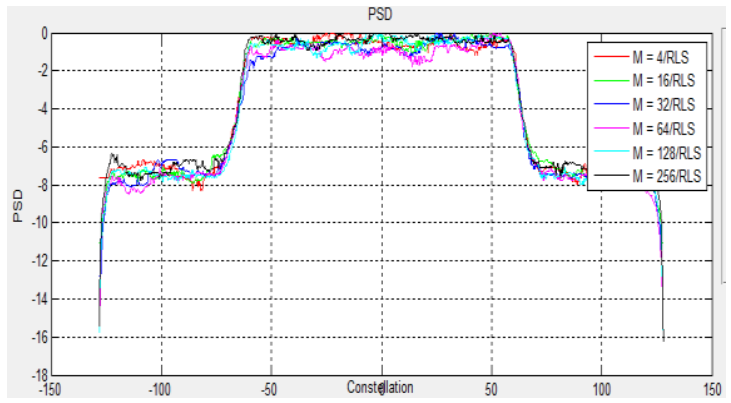

Fig. 9: Coefficient Estimation (PDF) for RLS Algorithm for Different Values of Constellation M-Ary=4, 16, 32, 64, 128, 256.

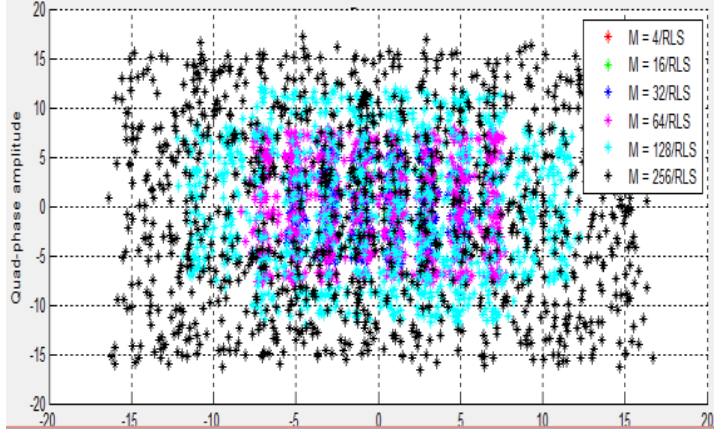

Fig. 10: Coefficient Estimation (Quad-Phase Amplitude) for RLS Algorithm for Different Values of Constellation M-Ary=4, 16, 32, 64, 128, 256.

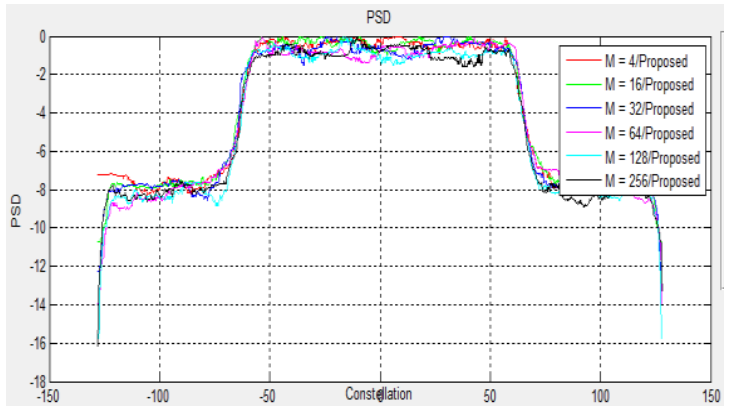

Fig. 11: Coefficient Estimation (PDF) For Proposed Algorithm for Different Values of Constellation M-Ary=4, 16, 32, 64, 128, 256.

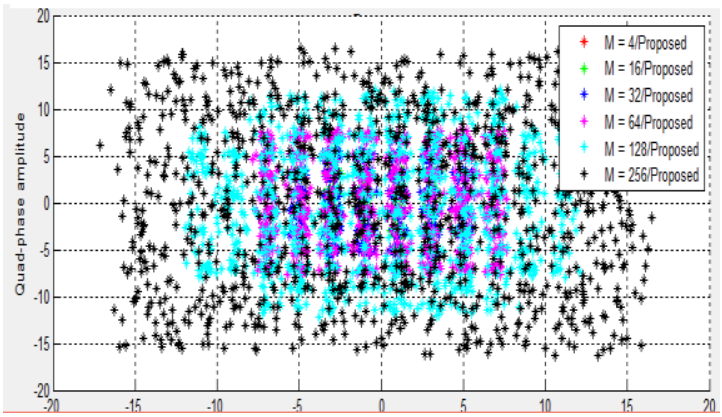

Fig. 12: Coefficient Estimation (Quad-Phase Amplitude) for Proposed Algorithm for Different Values of Constellation M-Ary=4, 16, 32, 64, 128, 256.

Table 2: Coefficient Estimation: for LMS $-\mathrm{K}=2$

\begin{tabular}{llll}
\hline \multirow{2}{*}{ Constellation M-ary } & ACPR & & \multirow{2}{*}{ EVM } \\
\hline 4 & L & H & \\
16 & 29.5673 & 28.3452 & -29.1502 \\
32 & 36.0264 & 36.7817 & -29.1063 \\
64 & 37.2101 & 38.37 & -29.1598 \\
128 & 41.8945 & 42.8825 & -29.0589 \\
256 & 47.3237 & 45.1915 & -28.9798 \\
\hline
\end{tabular}

Table 3: Coefficient Estimation: for RLS $-\mathrm{K}=2$

\begin{tabular}{llll}
\hline $\begin{array}{l}\text { Constellation } \\
\text { M-ary }\end{array}$ & ACPR & H & EVM \\
\hline 4 & 29.1988 & 28.882 & -30.2078 \\
16 & 34.7955 & 33.0891 & -29.9774 \\
32 & 34.9433 & 38.5547 & -30.0184 \\
64 & 42.3061 & 41.9217 & -30.2385 \\
128 & 44.1697 & 43.9847 & -30.2213 \\
256 & 46.7548 & 48.1497 & -30.093 \\
\hline
\end{tabular}

Table 4: Coefficient Estimation: For Proposed $-\mathrm{K}=2$

\begin{tabular}{llll}
\hline \multirow{2}{*}{ Constellation M-ary } & ACPR & \multirow{2}{*}{ EVM } \\
\hline 4 & L & H & \\
16 & 27.9781 & 25.7587 & -30.8544 \\
32 & 35.9127 & 37.0484 & -30.5935 \\
64 & 34.8564 & 37.4537 & -30.9674 \\
128 & 40.0128 & 41.7318 & -30.9903 \\
256 & 43.6804 & 41.5822 & -30.9941 \\
\hline
\end{tabular}




\section{Conclusion and future direction}

In this manuscript, we recommend an all-purpose method to derive the orthonormal basis functions that can significantly alleviate the numerical instability problem in coefficients estimation. Also, by applying the orthonormal basis functions, we further develop an adaptive algorithm that exhibits a low computation complexity of LMS algorithm while retaining the quick convergence velocity of RLS algorithm. Via both simulation and experimental results, we validate the implemented LMS algorithm yet as efficient as RLS algorithm. An efficient technique for improving the power signal spectral estimation is intended to propose here. The technique aims at enhancing the spectral estimation accuracy of RLS algorithm along with resolving its drawbacks. Further, the recursion minimization function will be included in the RLS algorithm to minimize the computation complexity. The technique provides spectra with improved resolution and smoother characteristics over competitive spectral estimation techniques. The proposed technique will be implemented in MATLAB working platform, and the output performance will be evaluated.

\section{References}

[1] M. R. Ram, K. Sivani and K. A. Reddy, "Utilization of adaptivecoefficient estimation method for Motion artifacts reduction from photoplethysmographic signals," 2016 International Conference on Wireless Communications, Signal Processing and Networking (WiSPNET), Chennai, India, 2016, pp. 818-822. https://doi.org/10.1109/WiSPNET.2016.7566247.

[2] M. Choi, J. J. Oh and S. B. Choi, "Linearized Recursive Leas Squares Methods for Real-Time Identification of Tire-Road Friction Coefficient," in IEEE Transactions on Vehicular Technology, vol. 62, no. 7, pp. 2906-2918, Sept. 2013. https://doi.org/10.1109/TVT.2013.2260190.

[3] S. Sardellitti and S. Barbarossa, "Distributed RLS estimation for cooperative sensing in small cell networks," 2013 IEEE International Conference on Acoustics, Speech and Signal Processing, Vancouver, BC, 2013, pp. 5283-5287. https://doi.org/10.1109/ICASSP.2013.6638671.

[4] D. Kobayashi, T. Imura and Y. Hori, "Real-time coupling coefficient estimation and maximum efficiency control on dynamic wireless power transfer for electric vehicles," Emerging Technologies: Wireless Power (WoW), 2015 IEEE PELS Workshop on, Daejeon, 2015, pp. 1-6. https://doi.org/10.1109/WoW.2015.7132799.

[5] K. T. Wagner and M. I. Doroslovački, "Combination coefficients for fastest convergence of distributed LMS estimation," 2014 IEEE International Conference on Acoustics, Speech and Signal Processing (ICASSP), Florence, 2014, pp. 7218-7222. https://doi.org/10.1109/ICASSP.2014.6855001.

[6] D. Kobayashi, T. Imura and Y. Hori, "Real-time coupling coefficient estimation and maximum efficiency control on dynamic wireless power transfer using secondary DC-DC converter," Industrial Electronics Society, IECON 2015 - 41st Annual Conference of the IEEE, Yokohama, 2015, pp. 004650-004655. https://doi.org/10.1109/IECON.2015.7392825.

[7] I. Umay, B. Fidan and M. R. Yüce, "Endoscopic capsule localization with unknown signal propagation coefficients," Advanced Robotics (ICAR), 2015 International Conference on, Istanbul, 2015, pp. 224229. https://doi.org/10.1109/ICAR.2015.7251460.

[8] G. Kedar-Dongarkar and M. Das, "Vehicle parameter estimation using nested RLS algorithm," 2013 IEEE 56th International Midwest Symposium on Circuits and Systems (MWSCAS), Columbus, $\mathrm{OH}$, 2013, pp. 404-407. https://doi.org/10.1109/MWSCAS.2013.6674671.

[9] B. K. Das and M. Chakraborty, "A new diffusion sparse RLS algorithm with improved convergence characteristics," 2016 IEEE International Symposium on Circuits and Systems (ISCAS), Montreal, QC, 2016, pp. 2651-2654. https://doi.org/10.1109/ISCAS.2016.7539138. 